\title{
Cross helicity at the solar surface by simulations and observations
}

\author{
G. Rüdiger ${ }^{1}$, M. Küker ${ }^{1}$, and R. S. Schnerr ${ }^{2}$ \\ ${ }^{1}$ Leibniz-Institut für Astrophysik Potsdam, An der Sternwarte 16, 14482 Potsdam, Germany \\ e-mail: [gruediger;mkueker] @aip.de \\ 2 Dept. of Astronomy, Stockholm University, Alba Nova University Center, 10691 Stockholm, Sweden
}

Received 22 March 2012 / Accepted 24 July 2012

\begin{abstract}
A result of the quasilinear mean-field theory for driven magnetohydrodynamic (MHD) turbulence is that the observed cross helicity $\langle\boldsymbol{u} \cdot \boldsymbol{b}\rangle$ may directly yield the magnetic eddy diffusivity $\eta_{\mathrm{T}}$ of the quiet Sun. In order to model the cross helicity at the solar surface, magnetoconvection under the presence of a vertical large-scale magnetic field is simulated with the nonlinear MHD code Nirvana. The very robust result of the calculations is that $\left\langle u_{z} b_{z}\right\rangle \simeq 2\langle\boldsymbol{u} \cdot \boldsymbol{b}\rangle$ independent of the applied magnetic field amplitude. The correlation coefficient for the cross helicity is about $10 \%$. Of similar robustness is the finding that the rms value of the magnetic perturbations exceeds the mean-field amplitude (only) by a factor of five. The characteristic helicity speed $u_{\eta}$ as the ratio of the eddy diffusivity and the density scale height for an isothermal sound velocity of $6.6 \mathrm{~km} \mathrm{~s}^{-1}$ prove to be $1 \mathrm{~km} \mathrm{~s}^{-1}$ for weak fields. This value coincides well with empirical results obtained from the data of the Hinode satellite and the Swedish 1-m Solar Telescope (SST) providing the crosshelicity component $\left\langle u_{z} b_{z}\right\rangle$. Both simulations and observations thus lead to the numerical value of $\eta_{\mathrm{T}} \simeq 10^{12} \mathrm{~cm}^{2} / \mathrm{s}$ as characteristic for the surface of the quiet Sun.
\end{abstract}

Key words. convection - magnetohydrodynamics (MHD) - Sun: granulation - Sun: surface magnetism

\section{Introduction}

It is not easy to measure the turbulent magnetic diffusivity $\eta_{\mathrm{T}}$ at the solar surface. This quantity determines the decay of magnetic structures with scales larger than those of the turbulence. Theoretically, the decay of the magnetic structures should depend on the relation of the magnetic field amplitude to the socalled equipartition value $B_{\mathrm{eq}}=\sqrt{\mu_{0} \rho\left\langle u^{2}\right\rangle}$ defined by the turbulence. This phenomenon is known as the effect of $\eta$-quenching, i.e., the suppression of the eddy diffusivity by the magnetic field.

The simplest realization of $\eta$-quenching at the solar surface can be given with two numbers. The decay of active regions after Schrijver \& Martin (1990) can be understood with an eddy diffusivity of $10^{12} \mathrm{~cm}^{2} / \mathrm{s}$, while the decay of sunspots with their much stronger fields leads to $10^{11} \mathrm{~cm}^{2} / \mathrm{s}$ (Stix 1989). These values are smaller than the value of $3 \times 10^{12} \mathrm{~cm}^{2} / \mathrm{s}$, which results from the widely used formula $\eta_{\mathrm{T}} \sim c_{\eta} u_{\mathrm{rms}} \ell_{\text {corr }}$ with the tuning parameter $c_{\eta} \simeq 0.3$, the correlation length $\ell_{\text {corr }}$, and parameter values taken close to the surface. Up to now, there was no possibility to measure the turbulent diffusivity on the solar surface for the quiet Sun, where magnetic quenching of this quantity by large-scale magnetic fields is negligible.

Rüdiger et al. (2011) have shown that the combination of a vertical field with driven turbulence in a density-stratified medium leads to an anticorrelation of the cross helicity and the vertical large-scale field, i.e., $\langle\boldsymbol{u} \cdot \boldsymbol{b}\rangle=-\eta_{\mathrm{T}} B_{z} / H_{\rho}$ with $H_{\rho}$ as the scale height of the density. If both the cross helicity and the large-scale vertical field are known, then the ratio of the eddy diffusivity and the density scale can be computed. If also the density scale is known from calculated atmosphere models, then fluctuation measurements can be used to calculate the numerical value of the eddy diffusivity for weak fields because if the large-scale magnetic field has only a vertical component and the only vertical gradient is a consequence of the density stratification, then $\langle\boldsymbol{u} \cdot \boldsymbol{b}\rangle \simeq\left\langle u_{z} b_{z}\right\rangle$. The correlation of the vertical components of flow and field can empirically be obtained by both Doppler measurements and spectropolarimetry.

To estimate the value of the cross helicity, we assume a density scale height of $100 \mathrm{~km}$ and write the result in the form

$\frac{\langle\boldsymbol{u} \cdot \boldsymbol{b}\rangle}{B_{z}} \simeq-\frac{\eta_{12}}{H_{7}} \quad \mathrm{~km} \mathrm{~s}^{-1}$

where $H_{7}=H_{\rho} / 100 \mathrm{~km}$ and $\eta_{\mathrm{T}}=10^{12} \eta_{12} \mathrm{~cm}^{2} / \mathrm{s}$. With observations of the left hand side of (1) of about $1 \mathrm{~km} \mathrm{~s}^{-1}$, one would find $\eta_{\mathrm{T}}$ of order $10^{12} \mathrm{~cm}^{2} / \mathrm{s}$. This is in line with the results from box simulations of mixed-polarity magnetic fields in the solar surface layer, where values between $10^{12} \mathrm{~cm}^{2} / \mathrm{s}$ and $3.4 \times 10^{12} \mathrm{~cm}^{2} / \mathrm{s}$ were found (Cameron et al. 2011). In the present paper, numerical simulations of stratified magnetoconvection and observational results are discussed, and the theory will be extended to include a vertical stratification of the turbulence intensity. Both the simulations and the observations lead to very similar results for the desired magnetic eddy diffusivity for the quiet Sun, exceeding the value given by (1) by a factor of (only) two.

A simple prediction of this theory is that the ratio (1) does not depend on the sign of the mean magnetic field, i.e., it does not vary from cycle to cycle and (for a dipolar field) from hemisphere to hemisphere. As a consequence, the sign of the cross helicity $\langle\boldsymbol{u} \cdot \boldsymbol{b}\rangle$ should vary from cycle to cycle and between the hemispheres. Zhao et al. (2011) indeed found indications for a variation from hemisphere to hemisphere in magnetograms and dopplergrams recorded by the Solar and Heliospheric Observatory's Michelson Doppler Imager in 2000, 2004, and 2007. 


\section{Mean-field electrodynamics}

Let $\boldsymbol{U}+\boldsymbol{u}$ and $\boldsymbol{B}+\boldsymbol{b}$ be the fluctuating velocity and magnetic field with the average values $\boldsymbol{U}$ and $\boldsymbol{B}$. The scalar correlation between the fluctuations of flow and field, i.e., the cross helicity $\langle\boldsymbol{u} \cdot \boldsymbol{b}\rangle$, is a pseudoscalar. In the same sense, the cross-correlation tensor $\left\langle u_{i} b_{j}\right\rangle$ is a pseudotensor. We are here only interested in its symmetric part

$H_{i j}=\frac{\left\langle u_{i} b_{j}\right\rangle+\left\langle u_{j} b_{i}\right\rangle}{2}$.

As we have shown, the tensor $H_{i j}$ can be finite in presence of a mean magnetic field $\boldsymbol{B}$ and for density-stratified fluids (Rüdiger et al. 2011). Consider these quantities as small enough so that expressions linear in the mean magnetic field influence of these quantities are sufficient. The same may hold for the shear, which influences the (radial) magnetic field components. It is then straightforward to formulate the relation

$$
\begin{aligned}
H_{i j}= & \alpha(\boldsymbol{G} \cdot \boldsymbol{B}) \delta_{i j}+\beta\left(G_{i} B_{j}+G_{j} B_{i}\right)+\gamma\left(B_{i, j}+B_{j, i}\right) \\
& +a(\boldsymbol{g} \cdot \boldsymbol{B})\left(U_{i, j}+U_{j, i}\right) \\
& +b\left(U_{i, l} B_{j}+U_{j, l} B_{i}\right) g_{l}+c\left(U_{i, l} g_{j}+U_{j, l} g_{i}\right) B_{l} \\
& +d\left(U_{l, i} B_{j}+U_{l, j} B_{i}\right) g_{l}+e\left(U_{l, i} g_{j}+U_{l, j} g_{i}\right) B_{l} .
\end{aligned}
$$

No other formations are possible linear in the mean field $\boldsymbol{B}$, the stratification vector $\boldsymbol{G}$, and the shear of the divergence-free mean flow $\boldsymbol{U}$. For the tensor components, we find

$H_{y z}=\beta g B_{y}+(a+b+c) g B_{z} U_{y, z}$

and

$H_{z z}=(\alpha+2 \beta) g B_{z}+2 e g B_{y} U_{y, z}$

if a box coordinate system $(x, y, z)$ for the latitudinal, azimuthal, and vertical direction is introduced. The $z$-axis is aligned with the stratification vector, i.e., it represents the radial direction in spherical geometry. The $x$ and $y$ coordinates denote the horizontal directions. Without shear, the correlation $H_{z z}$ measures the vertical magnetic field and the correlation $H_{y z}$ measures the azimuthal field. The correlations are also influenced by the shear $U_{y, z}$. With the shear included, finite values result for both the correlations (4) and (5), even when the field has only one component. For known values of the correlations, the coefficients and the vertical field of both the azimuthal field and the shear can be computed. We cannot, however, be sure that all the coefficients $a$....e must be nonzero. First test calculations of $H_{z z}$ under the presence of horizontal field and shear did not yield finite values of $e$ (Brandenburg, priv. comm.).

The turbulent flow is assumed anelastic, so that $\operatorname{div} \rho \boldsymbol{u}=0$. It is convenient to use the Fourier transformation of the momentum density $\boldsymbol{m}=\rho \boldsymbol{u}$, i.e.,

$\boldsymbol{m}(\boldsymbol{r}, t)=\int \hat{\boldsymbol{m}}(\boldsymbol{k}, \omega) \mathrm{e}^{\mathrm{i}(\boldsymbol{k} \cdot \boldsymbol{r}-\omega t)} \mathrm{d} \boldsymbol{k} \mathrm{d} \omega$

and likewise for the fluctuation of the magnetic field.

The spectral tensor of the momentum density that accounts for the stratification of the turbulence to the first-order terms reads

$$
\begin{aligned}
\left\langle\hat{m}_{i}(z, \omega) \hat{m}_{j}\left(z^{\prime}, \omega^{\prime}\right)\right\rangle=\delta\left(\omega+\omega^{\prime}\right) \frac{\hat{q}(k, \omega, \kappa)}{16 \pi k^{2}} & \\
& \times\left(\delta_{i j}-k_{i} k_{j} / k^{2}+\left(\kappa_{i} k_{j}-\kappa_{j} k_{i}\right) /\left(2 k^{2}\right)\right),
\end{aligned}
$$

where $k=\left(z-z^{\prime}\right) / 2, \kappa=z+z^{\prime}, \hat{q}$ is the Fourier transform of the local spectrum,

$q(k, \omega, \boldsymbol{r})=\rho^{2} E(k, \omega, \boldsymbol{r})=\int \hat{q}(k, \omega, \boldsymbol{\kappa}) \mathrm{e}^{\mathrm{i} \kappa \cdot \boldsymbol{r}} \mathrm{d} \boldsymbol{\kappa}$,

So that

$\left\langle u^{2}\right\rangle=\int_{0}^{\infty} \int_{0}^{\infty} E(k, \omega, \boldsymbol{r}) \mathrm{d} k \mathrm{~d} \omega$.

Derivation of the cross-correlation yields

$$
\begin{aligned}
H_{i j}= & \frac{1}{2} \eta_{\mathrm{T}}\left(G_{i} B_{j}+G_{j} B_{i}\right) \\
& -\left(\frac{3}{10} \eta_{\mathrm{T}}+\frac{2}{15} \hat{\eta}\right)\left(B_{j, i}+B_{i, j}\right),
\end{aligned}
$$

where $\boldsymbol{G}=\boldsymbol{\nabla} \log \rho$ is the gradient of density and

$\eta_{\mathrm{T}}=\frac{1}{3} \int_{0}^{\infty} \int_{0}^{\infty} \frac{\eta k^{2} E}{\omega^{2}+\eta^{2} k^{4}} \mathrm{~d} k \mathrm{~d} \omega$

$\hat{\eta}=\int_{0}^{\infty} \int_{0}^{\infty} \frac{\eta k^{2} \omega^{2} E}{\left(\omega^{2}+\eta^{2} k^{4}\right)^{2}} \mathrm{~d} k \mathrm{~d} \omega$

where $\eta$ is the molecular magnetic diffusivity. Both quantities remain finite in the high-conductivity limit.

From the cross-correlation tensor (10), the cross helicity $\langle\boldsymbol{u} \cdot \boldsymbol{b}\rangle=\eta_{\mathrm{T}}(\boldsymbol{G} \cdot \boldsymbol{B})$ is obtained. From Eq. (10) we find the slightly more complicated expression

$\left\langle u_{z} b_{z}\right\rangle=\eta_{\mathrm{T}} G B_{z}-\left(\frac{3 \eta_{\mathrm{T}}}{10}+\frac{2 \hat{\eta}}{15}\right)\left(2 \frac{\partial B_{z}}{\partial z}\right)$,

where $G=G_{z}$ is the only nonzero radial component of the density-stratification vector. Note the negativity of $G$. An upwards divergence of the mean field would reduce the effect of density stratification, but for uniform field components, the result is $\left\langle u_{z} b_{z}\right\rangle=\langle\boldsymbol{u} \cdot \boldsymbol{b}\rangle$.

A real difference between both correlation expressions is, however, due to a possible gradient $\boldsymbol{G}^{\prime}$ of the turbulence intensity $u_{\mathrm{rms}}$. One easily finds that for vertical fields the turbulence intensity gradient $G^{\prime}$ enters expressions for the correlations such as

$\langle\boldsymbol{u} \cdot \boldsymbol{b}\rangle=\left(G+\frac{1}{2} G^{\prime}\right) \eta_{\mathrm{T}} B_{z}, \quad\left\langle u_{z} b_{z}\right\rangle=\left(G+\frac{3}{10} G^{\prime}\right) \eta_{\mathrm{T}} B_{z}$.

In the bulk of the convection zone, $G^{\prime}=G_{z}^{\prime}$ is positive while $G$ is negative. Hence, $\left|\left\langle u_{z} b_{z}\right\rangle\right|>|\langle\boldsymbol{u} \cdot \boldsymbol{b}\rangle|$ for positive $B_{z}$, which is confirmed by the presented simulations (see below).

By elimination of $G^{\prime}$ one finds

$\frac{5}{2}\left\langle u_{z} b_{z}\right\rangle-\frac{3}{2}\langle\boldsymbol{u} \cdot \boldsymbol{b}\rangle=-\frac{\eta_{\mathrm{T}}}{H_{\rho}} B_{z}$.

The magnetic eddy diffusivity can thus be determined if the LHS of (15) is calculated from magnetoconvection simulations when the density scale height $H_{\rho}$ is known from numerical models of the solar atmosphere. As only the correlation $\left\langle u_{z} b_{z}\right\rangle$ can directly be observed, one needs a numerical model for the application of the LHS of (15) to derive the eddy diffusivity at the solar surface. 


\section{Numerical simulations}

We perform simulations for a number of different parameter combinations. These parameters include the strength of the imposed vertical field $B_{z}$, the viscosity $v$, and the magnetic diffusivity coefficient $\eta$.

The numerical simulations are done using the Nirvana code, which uses a conservative finite difference scheme (Ziegler 2004). We use Cartesian coordinates. The code solves the equation of motion,

$$
\begin{aligned}
& \frac{\partial(\rho \boldsymbol{u})}{\partial t}+\nabla \cdot\left[\rho \boldsymbol{u} \boldsymbol{u}+\left(p+\frac{1}{8 \pi}|\boldsymbol{B}|^{2}\right) I-\frac{1}{4 \pi} \boldsymbol{B} \boldsymbol{B}\right]= \\
& \nabla \cdot \tau+\rho \boldsymbol{f}_{\mathrm{e}},
\end{aligned}
$$

the induction equation,

$$
\frac{\partial \boldsymbol{B}}{\partial t}-\nabla \times(\boldsymbol{u} \times \boldsymbol{B}-\eta \nabla \times \boldsymbol{B})=0,
$$

the equation of mass conservation,

$$
\frac{\partial \rho}{\partial t}+\nabla \cdot(\rho \boldsymbol{u})=0
$$

and the equation of energy conservation,

$$
\begin{gathered}
\frac{\partial e}{\partial t}+\nabla \cdot\left[\left(e+p+\frac{1}{8 \pi}|\boldsymbol{B}|^{2}\right) \boldsymbol{v}-\frac{1}{4 \pi}(\boldsymbol{u} \cdot \boldsymbol{B}) \boldsymbol{B}\right]= \\
\nabla \cdot\left[\boldsymbol{u} \tau+\frac{\eta}{4 \pi} \boldsymbol{B} \times(\nabla \times \boldsymbol{B})-\boldsymbol{F}_{\text {cond }}\right]+\rho \boldsymbol{f}_{\mathrm{e}} \cdot \boldsymbol{u} .
\end{gathered}
$$

In Eqs. (16) and (19), $\boldsymbol{f}_{\mathrm{e}}$ is the (external) gravity force and

$$
\tau=v\left(\nabla \boldsymbol{u}+(\nabla \boldsymbol{u})^{\mathrm{T}}-\frac{2}{3}(\nabla \cdot \boldsymbol{u}) I\right)
$$

the viscous stress tensor. The total energy density is the sum of the thermal, kinetic, and magnetic energy density:

$$
e=\epsilon+\frac{\rho}{2} \boldsymbol{u}^{2}+\frac{1}{2 \mu} \boldsymbol{B}^{2}
$$

We assume an ideal gas with a constant mean molecular weight $\mu=1$. The thermal energy density is then

$$
\epsilon=\rho T \frac{\mathcal{R}}{\gamma-1} \text {. }
$$

with $\gamma=c_{p} / c_{v}=5 / 3$.

The gas is heated from below and kept at a fixed temperature at the top of the simulation box. Periodic boundary conditions apply at the horizontal boundaries. A homogeneous vertical magnetic field is applied. The upper and lower boundaries are impenetrable and stress-free.

The simulation volume is a rectangular box. The stratification is along the $z$-coordinate, and it is piecewise polytrophic, with the polytrophic index chosen such that the hydrostatic equilibrium state is convectively stable in the lower and unstable in the upper half of the simulation box. In the following, $p$ denotes gas pressure, $\rho$ mass density, $T$ temperature, $g$ gravity, $\kappa$ thermal conductivity, and $c_{p}$ the specific heat capacity at constant pressure.

The gas is initially in hydrostatic equilibrium, i.e.,

$$
\frac{\partial p}{\partial z}+\rho g=0
$$
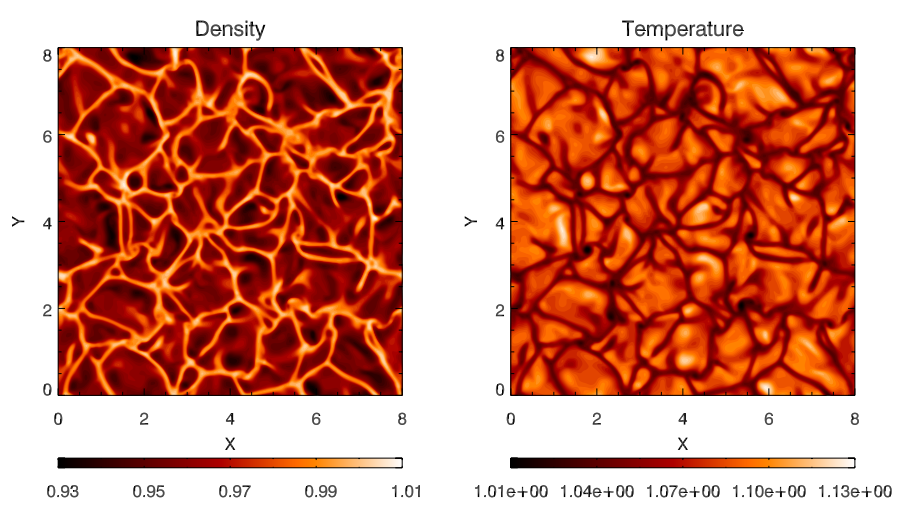

Fig. 1. Fluctuations of density and temperature in the upper part of the unstable layer at $z=-0.05$ for $\mathrm{Ra}=10^{7}$ and $B_{0}=1$.

where $g=$ const., and the heat flux through the box is vertical and constant,

$F_{0}=-\kappa \frac{\partial T}{\partial z}=$ const.

The equation of state is that for an ideal gas. The heat conductivity is constant in the upper and lower layer, respectively, but its values differ between the two layers.

In the dimensionless units, the size of the simulation box is $8 \times 8 \times 2$ in the $x, y$, and $z$ directions, respectively. The numerical resolution is $512 \times 512 \times 128$ grid points. The stratification of density, pressure, and temperature is piecewise polytrophic, as described in Ziegler (2002). Similar setups have been used by Cattaneo et al. (1991), Brummell et al. (1996), Brandenburg et al. (1996), Chan (2001) and Ossendrijver et al. (2001). The initial state is in hydrostatic equilibrium but convectively unstable in the upper half of the box. The $z$ coordinate is negative in our setup, with $z=0$ at the upper boundary. The stable layer thus extends from $z=-2$ to $z=-1$, the unstable layer from $z=-1$ to $z=0$. The density varies by a factor 5 over the depth of the box, i.e., the density scale height is 1.2.

Figure 1 shows snapshots of the fluctuations of density and temperature for $\mathrm{Ra}=10^{7}$ in a horizontal plane close to the upper boundary. The density is increased at the boundaries of the convection cells and decreased at the center. The opposite is true for the temperature, which is highest at the center of a convection cell and lowest at the boundaries. Vertical velocity is positive, i.e. upwards, at the center and negative, i.e. downwards, at the boundaries. The magnetic field is strongly concentrated in a few small patches which coincide with cell corners, where the gas horizontal flow converges and the vertical flow is downwards.

The initial magnetic field is vertical and homogeneous. We run the simulations until a quasistationary state evolves. Our control parameters are the heat conduction coefficient $\kappa$ and the Prandtl number $\operatorname{Pr}=v / \kappa$. Convection sets in if the Rayleigh number

$\mathrm{Ra}=\frac{\rho g c_{P} d^{4}}{T \kappa \nu}\left(\frac{\mathrm{d} T}{\mathrm{~d} z}-\frac{g}{c_{P}}\right)$

with the density $\rho$, the specific heat capacity $\mathrm{c}_{P}$, the gravity force $g$, and the length scale $d$, exceeds a critical value. The length scale is defined by the depth of the convectively unstable layer, i.e., $d=1$. After (1), the correlations and the mean magnetic field always have opposite signs. This has also been confirmed numerically. For positive values of the mean magnetic field $B_{z}$, the cross helicity is negative in the unstably stratified 

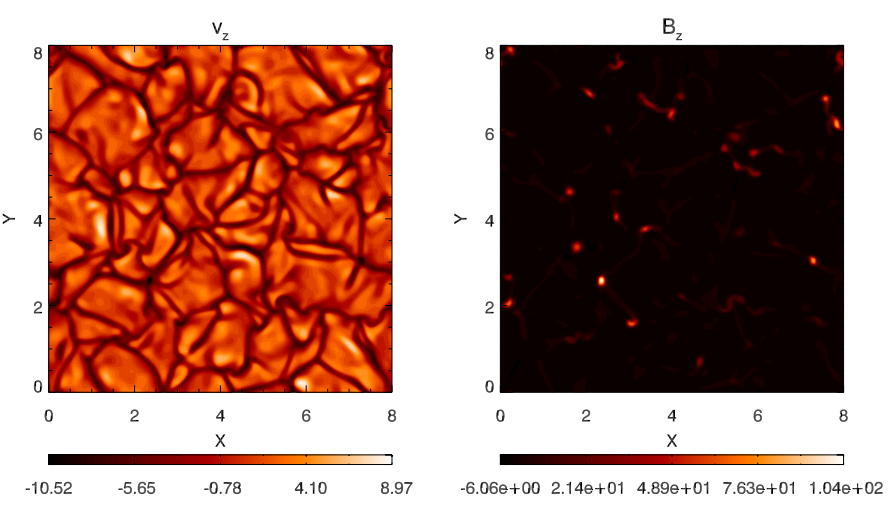

Fig. 2. The same as in Fig. 1 but for the fluctuations of the vertical flow and the vertical field.
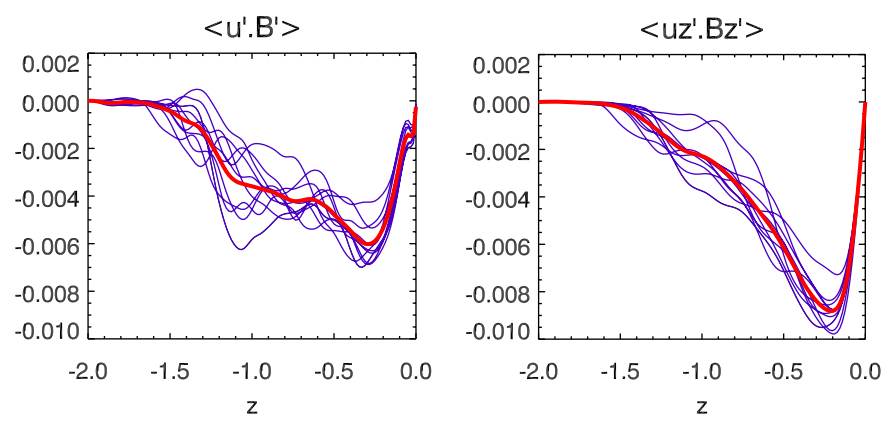

Fig. 3. Numerical values for the cross helicity $\langle\boldsymbol{u} \cdot \boldsymbol{b}\rangle($ left $)$ and the coefficient $\left\langle u_{z} b_{z}\right\rangle$ (right) for weak magnetic field $B_{z}=10^{-3}$. The blue lines denote individual snapshots, and the red lines average over the snapshots shown.
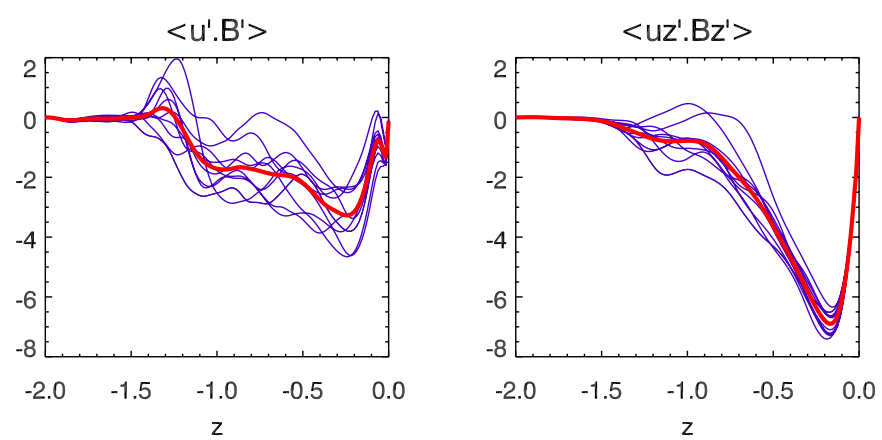

Fig. 4. The same as in Fig. 3 but for $B_{0}=1$.

layer. If the field polarity is reversed and everything else is left unchanged the cross-correlation becomes positive with the same amplitude.

The velocity field, which is measured in units of $c_{\mathrm{ac}} / 100$, shows the asymmetry between upwards and downwards motion as characteristic of convection in stratified media. The downwards motion is concentrated at the boundaries of the convection cells and particularly at the corners. The upwards motion fills the interior of the convection cells (see Fig. 2). As it covers a much larger area, the gas motion is considerably slower than in the concentrated downdrafts. The magnetic field shows a similar pattern. The vertical field is concentrated in the areas with downwards motion and weak in the areas with upwards motion. This is the result of field advection, as the total vertical magnetic flux is conserved.

Figures 3 and 4 hold for $\mathrm{Ra}=10^{7}$ and for weak and strong magnetic fields. The value of both the Prandtl number and the magnetic Prandtl number is 0.1 . The left diagram shows the horizontal average of the cross helicity as a function of the depth, and the right diagram shows the same for the correlation of the vertical components, $\left\langle u_{z} b_{z}\right\rangle$. There is a difference between the two quantities, with the vertical component actually being twice the cross helicity. Equation (15) can thus be written as

$\frac{\left\langle u_{z} b_{z}\right\rangle}{B_{z}} \simeq-\frac{4}{7} u_{\eta}$

with

$u_{\eta}=\frac{\eta_{\mathrm{T}}}{H_{\rho}}$.

The correlations do not vanish abruptly at the bottom of the unstable layer because of overshoot, which affects the upper half of the stable layer. The correlations there are positive and much smaller than in the unstable layer.

The results in Fig. 3 are given in arbitrary units defined by the code. Velocities are given in units of $c_{\mathrm{ac}} / 100$ with the isothermal speed of sound $c_{\mathrm{ac}}$. With an approximate value of $c_{\mathrm{ac}} \simeq$ $6.6 \mathrm{~km} \mathrm{~s}^{-1}$ at the optical depth $\tau=1$ of the Sun, the simulations lead to the cross-correlation velocity $\left\langle u_{z} b_{z}\right\rangle / B_{z} \simeq-9$ in units of $0.066 \mathrm{~km} \mathrm{~s}^{-1}$ (Fig. 3, right), i.e., after (26)

$u_{\eta} \simeq 1.04 \mathrm{~km} \mathrm{~s}^{-1}$.

This value depends only slightly on the magnetic field amplitude for weak fields. For the much stronger magnetic field, Fig. 4 (right) yields the slightly smaller value of $0.81 \mathrm{~km} \mathrm{~s}^{-1}$.

A characteristic velocity results as the cross-correlation velocity

$U_{\mathrm{c}}=\frac{|\langle\boldsymbol{u} \cdot \boldsymbol{b}\rangle|}{B_{z}}$.

Using the maximal values in Fig. 3 (left), we find $U_{\mathrm{c}} \simeq 6$ in units of $c_{\mathrm{ac}} / 100$. Hence, the simulations lead to the cross-correlation velocity $U_{\mathrm{c}} \simeq 0.4 \mathrm{~km} \mathrm{~s}^{-1}$. For the $B_{z}=1$ case (Fig. 4, left), we find $U_{\mathrm{c}} \simeq 3$ in units of $c_{\mathrm{ac}} / 100$ or $0.2 \mathrm{~km} \mathrm{~s}^{-1}$, respectively.

It also makes sense to normalize the cross-correlation in the form

$c_{\eta}=-\frac{\langle\boldsymbol{u} \cdot \boldsymbol{b}\rangle}{B_{z} \sqrt{\left\langle u^{2}\right\rangle}}$,

which is the ratio of the cross-correlation velocity (29) and the rms velocity of the turbulence. Its numerical value does not depend on the internal units of the code, so that $c_{\eta}$ is a general and basic result of the simulations. Close to the surface, the maximal numerical value is $c_{\eta} \simeq 0.6$ for $B_{z}=10^{-3}$ and $c_{\eta} \simeq 0.3$ for $B_{z}=1$. Test calculations for various magnetic fields over many orders of magnitudes show this value as almost uninfluenced by the magnetic-field suppression. Resulting from the overshoot phenomenon at the bottom of the unstable layer, small negative values always appear there. The correlation coefficient

$c=\frac{|\langle\boldsymbol{u} \cdot \boldsymbol{b}\rangle|}{\sqrt{\left\langle u^{2}\right\rangle} \sqrt{\left\langle b^{2}\right\rangle}}$,

for the cross helicity is much smaller than (30) as always

$\frac{\left\langle b^{2}\right\rangle}{B_{z}^{2}} \simeq 50$

similar to the result of Ossendrijver et al. (2001). The relation (31) proves to be true for all amplitudes of the mean magnetic field between $10^{-5}$ and 0.1 . One finds for all calculations 

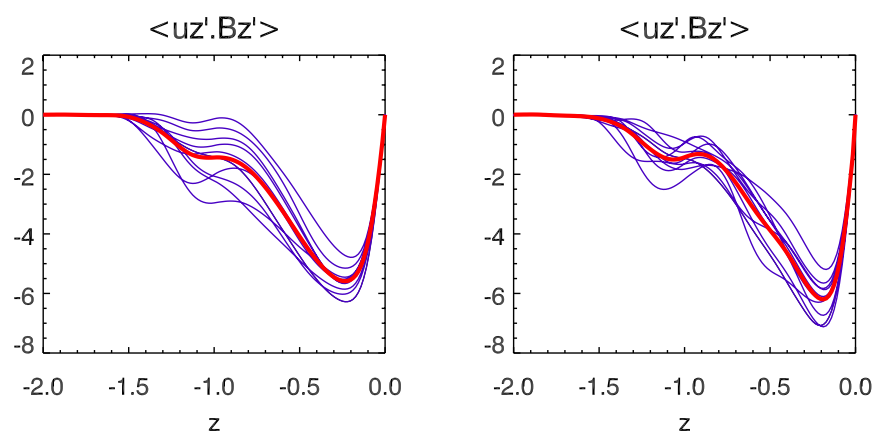

Fig. 5. The same as in the right part of Fig. 4 but with reduced numerical resolution of $128 \times 128 \times 128($ left $)$ and $256 \times 256 \times 128($ right $)$.
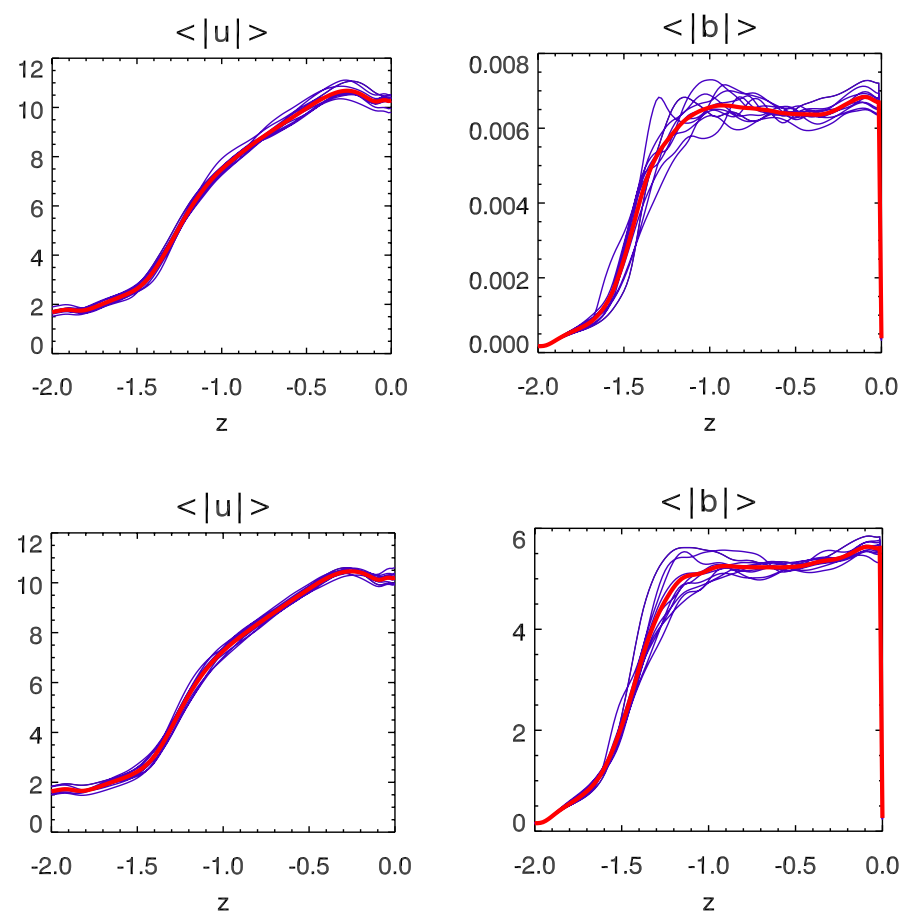

Fig. 6. Numerical values for $u_{\mathrm{rms}}$ (left) and $b_{\mathrm{rms}}$ (right) for weak magnetic field, $B_{z}=10^{-3}$ (top) and strong magnetic field, $B_{z}=1$ (bottom). Only the magnetic fluctuations depend on the background field amplitude.

a characteristic correlation coefficient $c \simeq 0.1$. The $B_{z}=1$ case shows the beginning of the suppression of the fluctuations by the mean magnetic field, which occurs at large values of $B_{z}$, resulting in a smaller value of 25 for $\left\langle b^{2}\right\rangle / B_{z}^{2}$.

During the simulations, there are significant temporal fluctuations. The convective instability initially grows exponentially until its saturation, when the system settles in a statistically steady state, but the cross helicity still shows some variations. We therefore average over a certain number of snapshots, typically ten. To test how much the results depend on the numerical resolution, we rerun the $B_{z}=1$ case at the lower resolutions of $256 \times 256 \times 128$ and $128^{3}$. Figure 5 shows $\left\langle u_{z} b_{z}\right\rangle$ from those runs. There is a weak dependence on resolution, with higher resolution leading to larger values.

Figure 6 contains all the information about the kinetic and magnetic energies of the magnetoconvection. The rms value of the velocity is hardly influenced by the large-scale magnetic field. In physical units, we find an averaged value of $u_{\mathrm{rms}} \simeq$ $0.1 c_{\mathrm{ac}} \simeq 0.66 \mathrm{~km} \mathrm{~s}^{-1}$. In contrast, the magnetic energy strongly
Table 1. Results from the analysis of the SST and Hinode data.

\begin{tabular}{lccccc}
\hline \hline Dataset & $\begin{array}{c}\text { Resolution } \\
\mathrm{km}\end{array}$ & $\begin{array}{c}\left\langle u_{z} b_{z}\right\rangle \\
{\left[\mathrm{G} \mathrm{km} \mathrm{s}{ }^{-1}\right]}\end{array}$ & $\begin{array}{c}B_{z} \\
{[\mathrm{G}]}\end{array}$ & $\begin{array}{c}\left\langle u_{z} b_{z}\right\rangle / B_{z} \\
{\left[\mathrm{~km} \mathrm{~s}^{-1}\right]}\end{array}$ & $\begin{array}{c}u_{\eta} \\
{\left[\mathrm{km} \mathrm{s}^{-1}\right]}\end{array}$ \\
\hline Hinode & 230 & -1.04 & 2.55 & -0.41 & 0.71 \\
SST & 115 & -1.82 & 2.54 & -0.72 & 1.26 \\
SST $^{*}$ & 172 & -1.57 & 2.54 & -0.62 & 1.08 \\
SST $^{*}$ & 258 & -1.32 & 2.54 & -0.52 & 0.91 \\
SST $^{*}$ & 343 & -1.05 & 2.54 & -0.41 & 0.72 \\
\hline
\end{tabular}

Notes. The resolution is measured on the solar surface; the SST resolution is reduced by rebinning the data. ${ }^{(*)}$ These data have been rebinned.

depends on the applied magnetic field. In dimensionless units, it is $b_{\mathrm{rms}} / u_{\mathrm{rms}} \simeq 0.6 B_{z}$ in both cases, which leads to

$\frac{E_{\mathrm{mag}}}{E_{\text {kin }}} \simeq 3600 \frac{B_{z}^{2}}{\mu_{0} \rho c_{\mathrm{ac}}^{2}}$

in physical units. At the top of the convection zone, we find very small contributions of the magnetic energy for $B_{z}=1$ Gauss, while for 1000 Gauss there is almost equipartition.

\section{Observations}

It is difficult to empirically determine the cross helicity $\langle\boldsymbol{u} \cdot \boldsymbol{b}\rangle$ at the solar surface because it is hard to retrieve the horizontal flows and magnetic field components from observations. We have, however, the possibility to use the relation $\langle\boldsymbol{u} \cdot \boldsymbol{b}\rangle \approx 0.5\left\langle u_{z} b_{z}\right\rangle$, known from the above numerical simulations. The vertical flow speed and magnetic field component can be determined with much better accuracy. Then using Eq. (26), we can determine the cross-helicity velocity from the observations.

For this purpose, we have analysed two datasets containing observations of the quiet Sun at disk center, where the lineof-sight coincides with the local vertical. Data from the Crisp Imaging Solar Polarimeter (CRISP) instrument on the Swedish 1-m Solar Telescope (SST) cover the 6302.5 ̊ Fe I spectral line with 12 equidistant wavelength positions at $48 \mathrm{~m} \AA$ steps and a continuum point. They have a pixel scale of 0 ". 0592 and a total field-of-view of about $60^{\prime \prime} \times 60^{\prime \prime}$. The second dataset is from the spectropolarimeter on the Solar Optical Telescope (SOT) of Hinode. It covers both the 6301.5 and $6302.5 \AA$ Fe I lines, has a pixel scale of 0 ". 16 , and a total (scanned) field of view of $164^{\prime \prime} \times 328^{\prime \prime}$.

The line-of-sight velocity and magnetic field data for the Hinode observations were taken from the level two data products available online ${ }^{1}$. Magnetic field strengths have been converted to fluxes by taking the filling factor into account. The SST data were inverted using the lilia inversion code (Socas-Navarro 2001). Velocities were calibrated using the convective blueshift determined by de La Cruz Rodríguez et al. (2011). More details on these two datasets can be found in Schnerr \& Spruit (2011).

We show the results for these datasets in Table 1. The crosshelicity velocity $\left(u_{\eta}\right)$ as determined from the SST data is somewhat higher than that from the Hinode data. This is at least partly due to the lower resolution of Hinode as compared to the SST. If we rebin the SST data to a lower resolution, the cross-helicity velocity decreases (see Table 1) because the strongest fields and flows are smoothed out.

The coefficient $\left\langle B_{z}^{2}\right\rangle /\left\langle B_{z}\right\rangle^{2}$ from the Hinode and SST data is 521.3 and 163.5 respectively, which is larger than the value

${ }^{1}$ http://sot.lmsal.com/data/sot/level2dd 
of 50 found in the simulations. This indicates that the effective magnetic Reynolds number in the simulations is smaller than in the solar convection zone.

\section{Conclusions}

We have shown that nonrotating turbulence at the top of the solar convection zone under the influence of a vertical magnetic field forms a finite cross helicity. The only condition is the existence of a vertical stratification of density and/or turbulence intensity. While the effect would not appear within the Boussinesq approximation, it exists in the high-conductivity limit, i.e., for sufficiently large magnetic Reynolds numbers.

In our understanding, the cross helicity is anticorrelated to the mean radial magnetic field, i.e.,

$$
\langle\boldsymbol{u} \cdot \boldsymbol{b}\rangle \cdot B_{z}<0 .
$$

For an oscillating dipolar background field, the sign of the cross helicity differs for both hemispheres and also from cycle to cycle.

The theory can also be used to measure the magnetic diffusivity if the cross helicity is known by observations. In order to find the cross helicity, one has only to correlate observed flow fluctuations with observed magnetic fluctuations.

The anticorrelation (34) for density-stratified turbulence has been established by Rüdiger et al. (2011) for a model of numerically-driven turbulence. In the present paper, buoyancydriven magnetoconvection has been simulated in a box with the Nirvana code. We find that such a turbulence also fulfills the relation (34). The correlation coefficient (30) takes the value of 0.6 for the weak magnetic field $B_{z}=10^{-3}$ and 0.3 for the stronger field $B_{z}=1$. The ratio (32) of the magnetic fluctuations to the applied magnetic field is always of the order five.

We have also shown that for density-stratified turbulence the identity $\langle\boldsymbol{u} \cdot \boldsymbol{b}\rangle=\left\langle u_{z} b_{z}\right\rangle$ holds. So far, solar observations can only measure the correlation $\left\langle u_{z} b_{z}\right\rangle$. The numerical simulations, however, always lead to the result $\left\langle u_{z} b_{z}\right\rangle \simeq 2\langle\boldsymbol{u} \cdot \boldsymbol{b}\rangle$, so that the observed value of $\left\langle u_{z} b_{z}\right\rangle$ would overestimate the actual cross helicity by a factor of two. The reason is the vertical stratification of the turbulence intensity, which at the top of the convection zone is antiparallel to the density stratification. Hence, both the correlations $\left\langle u_{z} b_{z}\right\rangle$ and $\langle\boldsymbol{u} \cdot \boldsymbol{b}\rangle$ are reduced but not by the same amount.

With $\left\langle u_{z} b_{z}\right\rangle \simeq 2\langle\boldsymbol{u} \cdot \boldsymbol{b}\rangle$, the value of $u_{\eta}$ can be computed by using Eq. (26). The numerical simulations lead to $u_{\eta} \simeq 1 \mathrm{~km} \mathrm{~s}^{-1}$ and $u_{\eta} \simeq 0.8 \mathrm{~km} \mathrm{~s}^{-1}$ respectively for the two cases studied. This result is well confirmed by the observations that lead to values between $0.7 \mathrm{~km} \mathrm{~s}^{-1}$ (Hinode) and $1.3 \mathrm{~km} \mathrm{~s}^{-1}$ (SST). To estimate the value of the eddy diffusivity at the solar surface, we shall assume a density scale height of $100 \mathrm{~km}$ and find values close to $\eta_{\mathrm{T}} \simeq 10^{12} \mathrm{~cm}^{2} / \mathrm{s}$ for the eddy diffusivity at the surface of the quiet Sun.

Acknowledgements. We gratefully acknowledge Axel Brandenburg (Stockholm) for motivating discussions and numerical support.

\section{References}

Brandenburg, A., Jennings, R. L., Nordlund, Å., et al. 1996, JFM, 306, 325 Brummell, N. H., Hurlburt, N. E., \& Toomre, J. 1996, ApJ, 473, 494 Cameron, R., Vögler, A., \& Schüssler, M. 2011, A\&A, 533, A86

Cattaneo, F., Brummell, N. H., Toomre, J., Malagoli, A., \& Hurlburt, N. E. 1991, ApJ, 370, 282

Chan, K. L. 2001, ApJ, 548, 1102

de La Cruz Rodríguez, J., Kiselman, D., \& Carlsson, M. 2011, A\&A, 528, A113

Ossendrijver, M., Stix, M., \& Brandenburg, A. 2001, A\&A, 376, 713

Rüdiger, G., Kitchatinov, L. L., \& Brandenburg, A. 2011, Sol. Phys., 269, 3

Schnerr, R. S., \& Spruit, H. C. 2011, A\&A, 532, A136

Schrijver, C. J., \& Martin, S. F. 1990, Sol. Phys., 129, 95

Socas-Navarro, H. 2001, in Advanced Solar Polarimetry - Theory, Observation, and Instrumentation, ed. Sigwarth, ASPCS, 236, 487

Stix, M. 1989, The Sun: An Introduction (Berlin, Heidelberg, New York: Springer)

Zhao, M. Y., Wang, X. W., \& Zhang, H. Q. 2011, Sol. Phys., 270, 23

Ziegler, U. 2002, A\&A, 386, 331

Ziegler, U. 2004, J. Comput. Phys., 196, 393 